\title{
Una aproximación a los métodos mixtos a partir de las trayectorias migratorias de profesionales rumanos en Barcelona
}

\author{
A mixed method approach to the migratory trajectories of \\ Romanian professionals in Barcelona
}

\author{
Alisa Petroff \\ Universidad Autónoma de Barcelona \\ Miembro del CER-Migracions (UAB) \\ alisageorgeta.petroff@uab.cat (ESPAÑA)
}

Recibido: 06.112015

Aceptado: 29.11 .2016

\section{RESUMEN}

El presente artículo tiene dos objetivos principales: 1) destacar las contribuciones de los diseños mixtos de carácter secuencial en el estudio de las migraciones y 2) explicar las trayectorias de un perfil de inmigrantes poco común en el contexto español. El diseño incluye una encuesta online a 217 rumanos pertenecientes a una red de profesionales en el extranjero, 30 entrevistas biográficas y 30 gráficos de satisfacción vital a profesionales residentes en Barcelona. En cuanto a las aportaciones metodológicas, el artículo pone de manifiesto la utilidad de la encuesta online (y el análisis de clasificación) como herramienta para determinar la selección de los perfiles estudiados en la segunda etapa (cualitativa). La segunda aportación metodológica del artículo guarda relación con la manera en la que se han utilizado e imbricado los gráficos de satisfacción vital con el análisis de las entrevistas biográficas. Finalmente, la principal aportación teórica destaca la necesidad de superar las concepciones clásicas de las migraciones cualificadas entendidas como fugas de cerebros. Es por ello que se trae a colación la relevancia de las redes de cerebros a la vez que se cuestiona que la circularidad sea un fenómeno que se produzca de forma automática. En este sentido, la heterogeneidad de perfiles que se plantean retornar, permanecer o reemigrar, responde a una diversidad de razones para migrar y a incorporaciones laborales distintas dependiendo del tipo de recursos que estas personas son capaces de movilizar durante su migración. Así mientras aquéllos que llegan a través del traslado de una empresa multinacional cuentan con mallas de seguridad que 
les permiten plantearse proyectos migratorios abiertos, para los que responden al perfil de estudiantes internacionales, la institución se percibe como un trampolín para la incorporación sin descartar la reemigración. Finalmente, cuando la migración se produce por la fuerza de los factores de atracción y expulsión, la permanencia en España es la opción más viable.

\title{
PALABRAS CLAVE
}

Inmigración cualificada, inmigrantes rumanos, trayectorias migratorias, profesionales en España, métodos mixtos

\begin{abstract}
Two are the main aims of the paper: to highlight the contributions of sequential mixed method designs in the study of migrations, more specifically in the study of migratory trajectories of skilled Romanian professionals in Barcelona and explain the trajectories of a profile of immigrants that in the Spanish context must be understood from a double exceptionality. Based on a design that includes an online survey to 217 Romanians (belonging to a professional network abroad), 30 biographical interviews and 30 life charts satisfaction of Romanian professionals living in Barcelona, the article tackles the way mixed methods are shaped in the case of skilled profiles of migrants and the particularities of a profile that counts with a low social and academic visibility. As for the methodological contributions, the article reveals the usefulness of online survey (and cluster analysis) as a tool to determine the selection of the profiles studied in the second phase of the study (qualitative). The second methodological contribution links with the way life satisfaction charts may be combined with the analysis of biographical interviews. Finally, the main empirical contribution highlights the heterogeneous nature of the profile of skilled migrants, who develops strategies and different paths depending on the set of resources that mobilize before and especially after migrating. While those who migrate through the transfer of a multinational company count on a safety net that is the company itself, for those who correspond to the profile of international students, the institution is perceived as a launching platform that leads to the incorporation into the skilled labour market, even in times of economic crisis. Finally, when the meso level resources do not exist, the micro-level resources (social networks) are the one that explain successful careers.
\end{abstract}

\section{KEY WORDS}

Skilled migration, Romanian migration, migratory trajectories, professionals in Spain, mixed methods 


\section{INTRODUCCIÓN}

Aunque históricamente las perspectivas cuantitativas y cualitativas se presentan como paradigmas radicalmente diferentes, en las últimas décadas se han alzado numerosas voces que se manifiestan a favor de la convergencia, y que sugieren el uso conjunto de los métodos cuantitativos y cualitativos (Brannen, 2004). Mientras la fuerza de los métodos cuantitativos reside en la producción de datos generalizables al conjunto de la población; para los métodos cualitativos esa fortaleza radica en su capacidad para generar datos detallados y ricos (Steckler et al., 1992). Los beneficios teóricos y metodológicos obtenidos de dicha combinación van más allá de la suma de elementos anteriormente existentes (Verd y Lopez, 2008). Aunque la forma en la que se combinan los diferentes métodos ha generado diferente terminología, el término "métodos mixtos" se utiliza para denominar a aquellos diseños que buscan un elevado grado de integración entre las perspectivas cuantitativa y cualitativa (Verd y Lopez, 2008). En esta línea, los diseños de métodos mixtos incorporan varias estrategias cualitativas y cuantitativas en un solo proyecto de investigación que puede tener una orientación principal, tanto cuantitativa como cualitativa (Morse, 2003: 190).

Con estos elementos como punto de partida, uno de los objetivos del artículo es destacar las bondades de los diseños mixtos de carácter secuencial en el estudio de las migraciones, concretamente para el estudio de las trayectorias migratorias de rumanos altamente cualificados residentes en Barcelona $^{1}$. Si bien la utilización de los métodos mixtos en el estudio de las migraciones no constituye una novedad, el artículo plantea un diseño metodológico que incorpora los gráficos de satisfacción vital desde una perspectiva cualitativa. Además de la aportación metodológica, el artículo cuestiona que las migraciones cualificadas se sigan entendiendo desde la perspectiva de fuga de cerebro. En base al estudio de caso planteado, se muestra cómo la movilidad intra-europea se debe entender en clave de circulación donde cada vez hay una mayor prevalencia de redes (formales e informales) de cerebros. No obstante, esta movilidad y circulación no es intrínseca a los movimientos intra-europeos sino que las motivaciones pero, sobre todo, el tipo de incorporación en el mercado laboral de los países de destino acaban marcando dicha circularidad.

En cuanto a la elección del objeto de estudio, este se debe entender desde una doble excepcionalidad: a la excepcionalidad de los inmigrantes cualificados en España, se le añade la de los inmigrantes rumanos cualificados en este contexto. A partir del año 2000 el aumento de la demanda para cubrir puestos de baja cualificación, supone la llegada a España de un perfil de inmigrante poco cualificado o que experimenta sobrecualificación (Cachón, 2002, Parella 2003; Pajares, 2009; Viruela,

${ }^{1}$ El artículo presenta los principales hallazgos de la tesis doctoral: Las trayectorias laborales de los inmigrantes rumanos cualificados en Barcelona (Petroff, 2013). 
2007; Marcu, 2007, Reyneri y Fullin, 2008). A diferencia de países como Inglaterra, Estados Unidos o Canadá, la atracción de profesionales extranjeros no ha representado una prioridad en la agenda de los diferentes gobiernos españoles; si bien en el 2007 se crea la Unidad de Grandes Empresas y se aprueba la Ley 14/2013, de 27 de septiembre, de apoyo a los emprendedores y su internacionalización. El cambio legislativo y la agencia pretenden agilizar el flujo de trabajadores altamente cualificados a pesar de que las cifras oficiales de personas que entran en España a través de esta vía son bajas (12.000 permisos entre 2007 y 2011) (Gómez-Ventura, 2013).

A la excepcionalidad del perfil de migrantes cualificados en España se añade una segunda excepcionalidad: la relevancia cuantitativa de profesionales rumanos residentes en este país. Si bien, tradicionalmente, las migraciones procedentes de Rumanía se han identificado (especialmente en los contextos de los países del Sur) con la migración no cualificada, a partir de la entrada en la UE se produce un punto de inflexión que viene acompañado de una diversificación de los perfiles. Concretamente, esta diversificación supone el incremento de los flujos cualificados, con especial presencia de médicos, arquitectos, economistas, especialistas en informática (Alexe et al., 2011, Ferro, 2008). Además, estos movimientos se enmarcan en la lógica de la movilidad, tanto temporal como circular, de la nueva clase media emergente rumana, que busca desarrollar carreras profesionales y educativas en el espacio transnacional de la interconectada Europa (Csedo, 2009, Marcu, 2013, 2015).

En relación a la estructura del artículo, comprende cuatro apartados. Un primer apartado presenta el marco conceptual de las migraciones cualificadas y destaca los aportes teóricos del estudio de caso. En segundo lugar, se presenta el diseño metodológico (mixto) de la investigación y se detalla la manera en la que se han imbricado los diferentes métodos (cualitativos y cuantitativos). La tercera parte incluye la presentación de los resultados, primero de la parte cuantitativa (encuesta online a una red de profesionales rumanos en el extranjero) y de la parte cualitativa (entrevistas biográficas semi-estructurada y gráficos de satisfacción vital). El artículo concluye con un apartado de reflexiones finales que pretenden resumir y discutir los principales hallazgos metodológicos y empíricos.

\section{REDES DE CEREBROS Y PATRONES DE CIRCULARIDAD: SUPERANDO EL PARADIGMA DE LA FUGA DE CEREBROS}

El concepto fuga de cerebros surge en la literatura sobre inmigración en los años 1960, para referirse a la pérdida de personal cualificado europeo que emigra hacia Estados Unidos (Ackers, 2005; Horvat, 2004). El término goza de tanto éxito, que hasta la década de los años 1990 el debate de la inmigración cualificada se enmarca en la perspectiva del brain drain (Meyer, 2003). A finales de los años 1990, los enfoques basados en el estado-nación 
empiezan a perder relevancia. A partir de la nueva realidad marcada por la globalización (Khadria, 2001), es cada vez más frecuente residir en otros países, convirtiendo el flujo regular entre países, en la tónica general (Carr et al., 2005). La migración internacional, históricamente un proceso de un solo sentido, hoy en día, gracias al desarrollo de las tecnologías, representa una elección reversible (Solé at alt, 2016).

En este contexto, surgen perspectivas que incluyen una visión cosmopolita de la migración cualificada y que ponen énfasis en el impacto global del desarrollo, a la luz de nuevos procesos. Así, de esta visión internacional del fenómeno emerge el paradigma de la circularidad (Gaillard y Gaillard, 1997), que describe los movimientos de migrantes cualificados como policéntricos, circulares, temporales y basados en el intercambio entre países (Ackers, 2005; Patterson, 2005). Según el paradigma de la circularidad, el brain circulation supone el incremento de la migración temporal y el posterior retorno de los migrantes cualificados. No obstante, el retorno no está exento de dificultades y esta visión optimista de la circularidad, entendida como un triple win, queda ensombrecida por el choque cultural inverso que experimentan los retornados tras convivir durante años en sistemas democráticos, meritocráticos, etc (Ghatak, 2004). A esto se le añade que el retorno no siempre viene acompañado por una transferencia automática de las competencias adquiridas en el extranjero ya que las redes sociales son fundamentales para asegurar la incorporación laboral (Meyer, 2001).

A raíz de estas críticas, surgen diferentes conceptos que superan la necesidad de retornos permanentes y contemplan la migración cualificada desde la perspectiva de las redes de profesionales. Desde la perspectiva transnacional, se desarrollan conceptos como la opción de la diáspora (Horvat, 2004) o el brain networking (Ciumasu, 2007). Así, gracias a la configuración de redes formales e informales y a las facilidades de las tecnologías modernas, se generan intercambios de información y de conocimiento entre migrantes y países, sin la necesidad de que los retornos sean permanentes o físicos (Carr et al., 2005, Petroff, 2016b). Es más, cuando estas redes surgen a raíz de iniciativas gubernamentales o su desarrollo se promueve a través de programas, la eficacia de las mismas aumenta. Así, a través de estrategias coordinadas por los gobiernos de los países emisores se facilita e impulsa la transferencia de conocimiento y tecnología de unos países a otros (Patterson, 2005).

Si bien en este artículo destacamos el potencial de las redes de cerebros a partir del estudio de caso presentado (etapa cuantitativa), cuestionamos que la circularidad (física o virtual) se produzca de forma automática (etapa cualitativa). En este sentido, el paradigma de la movilidad (Hannam, Sheller and Urry, 2006) apunta que la proliferación de lugares y tecnologías genera que algunas personas experimenten movilidad mientras que otras experimenten inmovilidad. Así, ponemos de manifiesto que las razones para migrar y también el tipo de incorporación en el mercado laboral de los 
países de destino, marcan la forma en la que los migrantes proyectan sus perspectivas de futuro en clave de retorno, permanencia en el país de destino o reemigración. En el caso de aquellos migrantes que migran a través de traslado de empresa multinacional, las mallas de seguridad que ofrecen estas compañías permiten construir proyectos migratorios abiertos, donde tanto la permanencia como el retorno o la reemigración se enmarcan dentro de las oportunidades que ofrece la empresa. Si bien los rumanos que completan sus estudios de postgrado en España migran con la idea de retornar y capitalizar sus inversiones educativas, a medida que la incorporación laboral se consolida en clave ascendente, la permanencia o incluso la reemigración se convierten en las opciones más viables. Por último, aquellos que migran a España por la fuerza de los factores de atracción y expulsión, se proyectan a largo plazo en este país. Sus trayectorias migratorias disruptivas explican en gran medida esta decisión.

\section{ESTRATEGIA METODOLÓGICA}

La investigación se ha realizado en base a un diseño en fases secuenciales (Creswell, 2003). Así, los datos analizados en una primera fase han servido para mejorar la obtención de datos y análisis de la siguiente fase. En relación al orden, si tradicionalmente la investigación cualitativa se utiliza en las fases exploratorias del proceso de investigación, las perspectivas más recientes toman en consideración el uso de las encuestas en las fases preliminares del diseño para complementar la fase cualitativa (Bauer et al, 2000). Es precisamente ésta la secuencia de los dos métodos utilizados (Cuadro1).

Cuadro 1: Las fases del diseño mixto de la investigación

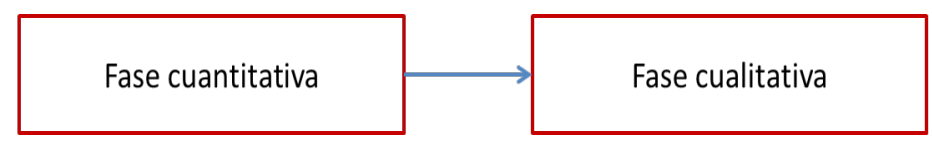

Recogida y análisis de datos de la
encuesta online a miembros
GRASP $\quad \begin{aligned} & \text { Entrevistas biográficas a } \\ & \text { rumanos cualificados en } \longrightarrow \begin{array}{l}\text { Interpretación } \\ \text { conjunta }\end{array}\end{aligned}$

Fuente: elaboración propia en base a la adaptación de Verd y Lopez (2008) 


\subsection{La fase cuantitativa: encuesta online a la red de profesionales GRASP}

En la fase cuantitativa, a través de la técnica de la encuesta online, se han identificado diferentes perfiles de rumanos cualificados con el objetivo de escoger uno y establecer el casillero tipológico de la fase cualitativa. Para ello, se ha utilizado la base de datos elaborada a partir de la encuesta online realizada a 217 miembros de la red de profesionales GRASP ${ }^{2}$, encuesta llevada a cabo de septiembre a diciembre 2010. La manera en la que se ha contactado con los encuestados ha sido a través del grupo que la organización tiene en la red social Facebook. Esta estrategia a la hora de acceder a los potenciales encuestados conlleva principalmente una limitación: dado que la muestra es autoselectiva, los resultados se han de entender exclusivamente como una aproximación a los diferentes perfiles de migrantes rumanos cualificados.

El cuestionario online incluye cuatro bloques temáticos y se han elegido 18 preguntas para el análisis: un primer bloque de preguntas explora la configuración del proyecto migratorio. Un segundo bloque contempla el papel que juegan las redes sociales en la constitución del proyecto migratorio. Un tercer bloque agrupa variables que hacen referencia al ámbito laboral en clave de trayectoria. Finalmente, el último bloque incluye variables sobre la participación en actividades de tipo comunitario, conjuntamente con otros rumanos.

En relación a la técnica de análisis, la primera fase de la investigación apuesta por el análisis de clasificación, que consiste en clasificar las unidades para obtener un conjunto de tipos que son la expresión de grupos de unidades homogéneas internamente y significativamente diferenciadas entre sí (Domínguez y López, 1996). Varios han sido los pasos seguidos a la hora de realizar el análisis de clasificación:

1. La selección de las variables, y un primer nivel de análisis descriptivo y exploratorio.

2. En base a las variables seleccionadas, se ha realizado el análisis de correspondencias múltiple, sin especificar el número de factores (utilizando el programa informático SPAD).

3. A partir de los factores que se han identificado, se han

${ }^{2}$ GRASP (Global Romanian Society of Young Professionals) es una organización sin ánimo de lucro que se institucionaliza en el año 2006. Su principal objetivo es conectar a rumanos cualificados que residen en diferentes partes del mundo y desarrollar proyectos en una variedad de ámbitos, con el propósito de generar impactos positivos en el país de origen. La solicitud para realizar la encuesta se envió a los 1020 personas que en aquel momento formaban parte del grupo de Facebook MyGRASP pidiendo que contesten solamente aquellos que residían en el extranjero ya que cabe mencionar que algunos de los miembros de esta organización desarrollan su actividad en Rumania o habían retornado en el momento de la encuesta. 
calculado los valores propios transformados para obtener el número óptimo de factores.

4. Posteriormente, se ha repetido el análisis de correspondencias múltiple, esta vez mencionando el número de dimensiones, a través de las cuales la varianza explicada es la óptima.

5. Una vez establecidos los factores de heterogeneidad y presentadas las características en relación a los respectivos ejes, se ha realizado el análisis de clasificación.

6. Tras el proceso de validación técnica, y tras el análisis de los datos del histograma de índices de nivel y el dendograma, el contenido final de grupos resultantes se ha expresado en tipos de la tipología (clusters). A través de estas tipologías se han clasificado a los individuos en base al número de grupos resultantes.

7. En la última etapa se han presentado los tres clusters identificados.

\subsection{La fase cualitativa: las entrevistas biográficas y los gráficos de satisfacción vital a rumanos cualificados residentes en Barcelona}

La fase cualitativa se realizó a través de la técnica de la entrevista biográfica semi-estructurada y los gráficos de satisfacción vital. En cuanto a la perspectiva biográfica, implica conocer significados y contextos de significados de lo individual, en tanto que parte de lo social, e indagar en las estructuras y normas sociales (Mallimaci y Giménez, 2006; Gaskell, 2000). Por otro lado, los gráficos de satisfacción vital no suponen una herramienta nueva en las ciencias sociales y tradicionalmente se han utilizado desde una perspectiva cuantitativa, sobre todo por disciplinas como la psicología. Sin embargo, en la presente investigación estos gráficos se abordan desde una perspectiva cualitativa. Así, al principio de la entrevista se le presenta al entrevistado el gráfico 1 y se le pide que dibuje su propia trayectoria educativa y profesional, valorando el grado de satisfacción con respecto a cada una de las etapas (el eje horizontal representa la edad del entrevistado, empezando desde los 14 años; el eje vertical corresponde al grado de satisfacción con respecto a cada una de las etapas). El objetivo de los gráficos consiste en interpretar cualitativamente e identificar, no solo a través del discurso, sino también gráficamente, hasta qué punto determinados acontecimientos pueden ser interpretados como puntos de inflexión, a diferencia de otros, que representan suaves transiciones a lo largo de la trayectoria. 


\section{Gráfico1: Modelo de gráfico de satisfacción vital:}

TRAYECTORIA EDUCATIVO-LABORAL

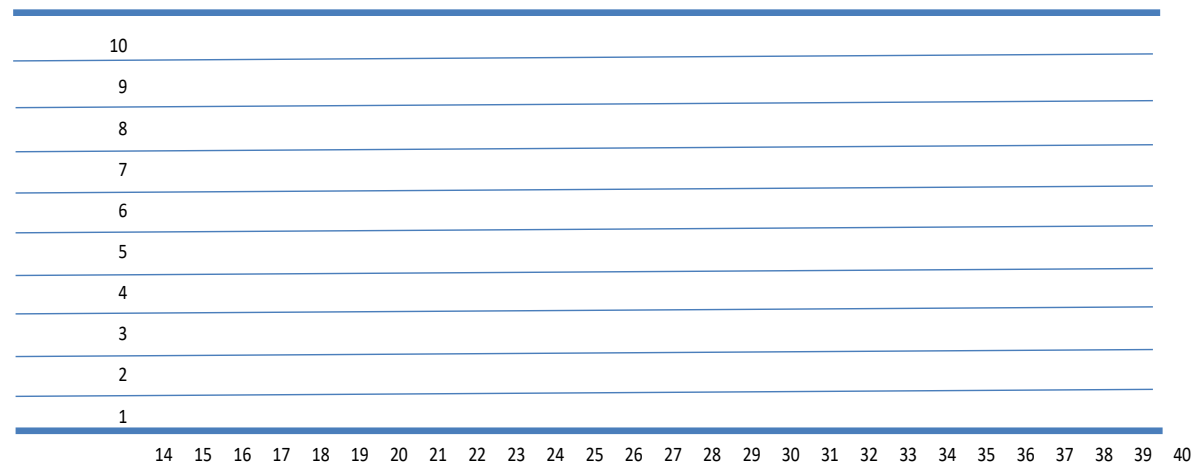

\section{Fuente: elaboración propia}

En cuanto a las características de la muestra cualitativa y las estrategias para realizar el trabajo de campo en Barcelona, cabe destacar los siguientes aspectos. Los contactos ${ }^{3}$ se han conseguido a través de la técnica de la bola de nieve. No obstante, se ha garantizado la heterogeneidad de los perfiles a partir de entrevistas a miembros de otras organizaciones de profesionales rumanos en el extranjero residiendo en Barcelona (GRASP, Liga de los estudiantes rumanos en el extranjero etc.), al igual que a personas que no pertenecen a ninguna red profesional. El trabajo de campo cualitativo se realizó de enero a mayo de 2012. Las entrevistas se llevaron a cabo en rumano, fueron grabadas, transcritas y posteriormente analizadas a través del programa Atlas.ti (cuadro 2).

${ }^{3}$ A partir de las entrevistas se ha desarrollado una red de profesionales rumanos online (Barcelona) Connecting Ideas reúne en noviembre de 2016 a 196 personas y logra ser un espacio de encuentro para la comunidad, de intercambio de información y recursos. 
Cuadro 2: Plantilla de los perfiles entrevistados y sus características

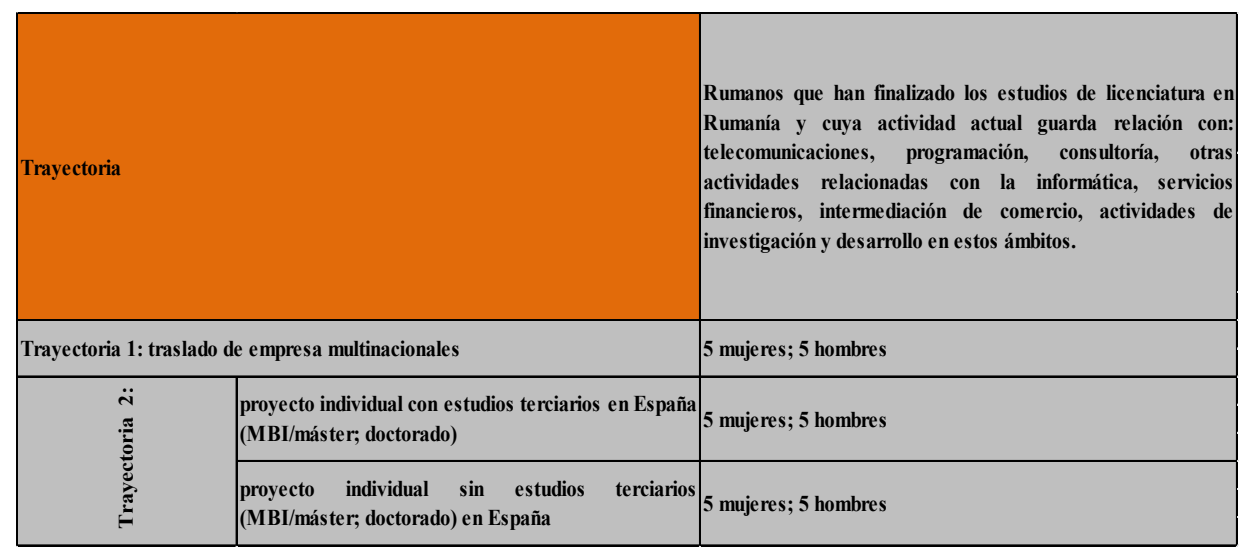

Fuente: elaboración propia ${ }^{4}$

Tal y como queda reflejado en el cuadro 2 y siguiendo los criterios de Gaskell (2000), se han realizado 30 entrevistas biográficas (15 a hombres y 15 a mujeres). La justificación de los sectores de actividad escogidos (IT, telecomunicación, servicios financieros etc.) tiene que ver con razones empíricas (que se presentan en la parte de hallazgos cuantitativos, concretamente en el cluster 3); pero también con razones teóricas: el auge, del sector de las nuevas tecnologías y servicios relacionados, explica, en gran medida, el aumento de las migraciones cualificadas (Iredale, 2001; BensonRea y Rawlinson, 2003).

\section{PRINCIPALES HALLAZGOS}

\subsection{Tipología de rumanos altamente cualificados en el extranjero}

En el primer análisis de correspondencias múltiples se incluyeron 18 variables, sin especificar el número de factores a tener en cuenta. Dado que el programa SPAD, por defecto, guarda diez variables factoriales y analiza los datos de cinco, el número de factores total obtenido de manera pesimista es 85. A partir de estos 85 factores y sus correspondientes valores propios, se calculan los valores propios transformados con el fin de obtener el número óptimo de factores y realizar un segundo análisis de correspondencias múltiple. Tras realizar los procedimientos necesarios, el número óptimo de

${ }^{4}$ Las actividades se corresponden a la Clasificación nacional de actividades económicas 2009, disponible en la página http://www.ine.es/daco/daco42/clasificaciones/cnae09/estructura.pdf 
factores se reduce a cuatro, asumiendo el $62,7 \%$ de la varianza explicada por estos cuatro factores.

Posteriormente, se realizó un segundo análisis de correspondencias múltiples. Esta vez se especificó el número de dimensiones (cuatro) que configuraron el análisis. La primera dimensión explica el 20,7\% de la varianza. El mayor peso lo tienen las siguientes variables: ocupación actual $(10,2)$; ocupación antes de emigrar $(9,8)$; situación antes de emigrar $(8,6)$; ámbito de trabajo $(8,2)$, edad $(8,3)$ y, en menor medida, el nivel de estudios obtenido en Rumanía $(6,2)$. Así, la primera dimensión opone a inmigrantes rumanos con una trayectoria profesional ya consolidada, frente a inmigrantes rumanos que responden al perfil de estudiantes internacionales.

En cuanto a la segunda dimensión $(19,8 \%)$, las variables con mayor poder explicativo son: si conocía a alguien en el país de destino $(10,7)$, relación con la mayoría de estas personas $(13,5)$ y el papel que juega esa persona en la decisión de emigrar $(11,6)$. A estas variables se les añaden, casi con la misma intensidad, variables como: país de residencia actual $(10,8)$, año de inmigración $(9,9)$, nivel de estudios obtenido en Rumanía $(9,7)$. La naturaleza de las variables que explican esta segunda dimensión permite identificar una emigración "post-comunista", de carácter permanente frente a una emigración "post-integración UE", circular y orientada hacia la formación, fruto de la movilidad tras la integración en la UE.

En la tercera dimensión (12\%) destacan tres variables: la participación en actividades comunitarias (16,7), tipo de actividades en las que participan $(16,9)$ y frecuencia de la actividad $(15,2)$. En menor medida las variables que hacen referencia a las razones para escogen el actual país de residencia $(5,3)$ y el lugar donde se han adquirido la mayoría de las competencias, habilidades y el know how $(5,6)$. Esta dimensión se distingue por el grado de vinculación cultural con el entorno. Por un lado, están aquellos profesionales que mantienen vínculos con la comunidad rumana, (como marco cultural de referencia) y, por otro lado, aquéllos que no participan en este tipo de actividades.

En la cuarta dimensión $(10,1 \%)$, vuelve a tener un papel relevante la variable referida a la ocupación actual $(11,2)$; así como la variable referida al ámbito de trabajo actual $(9,5)$. Con la misma intensidad destacan otras variables que hacen referencia al papel de las redes sociales: si conocía a alguien en el país de residencia $(7,6)$, relación con la mayoría de estas personas $(9,9)$ y papel de esas personas en la decisión de emigrar $(9,7)$. Teniendo en cuenta las características de esta dimensión, podemos vincularla al papel de los contactos y las redes sociales en la configuración del proyecto migratorio, así como a la actual posición que estos migrantes ocupan en el mercado laboral

Una vez establecidos estos cuatro factores de heterogeneidad y de haberlos caracterizado en relación a los respectivos ejes, se procedió a la realización del análisis de clasificación. Tras el proceso de validación técnica, y el análisis de los datos del histograma de índices de nivel y del 
dendograma, el contenido final de grupos resultantes se expresa en tres tipos de la tipología (Cuadro 3).

\section{Cuadro 3: Tres clusters de rumanos cualificados}

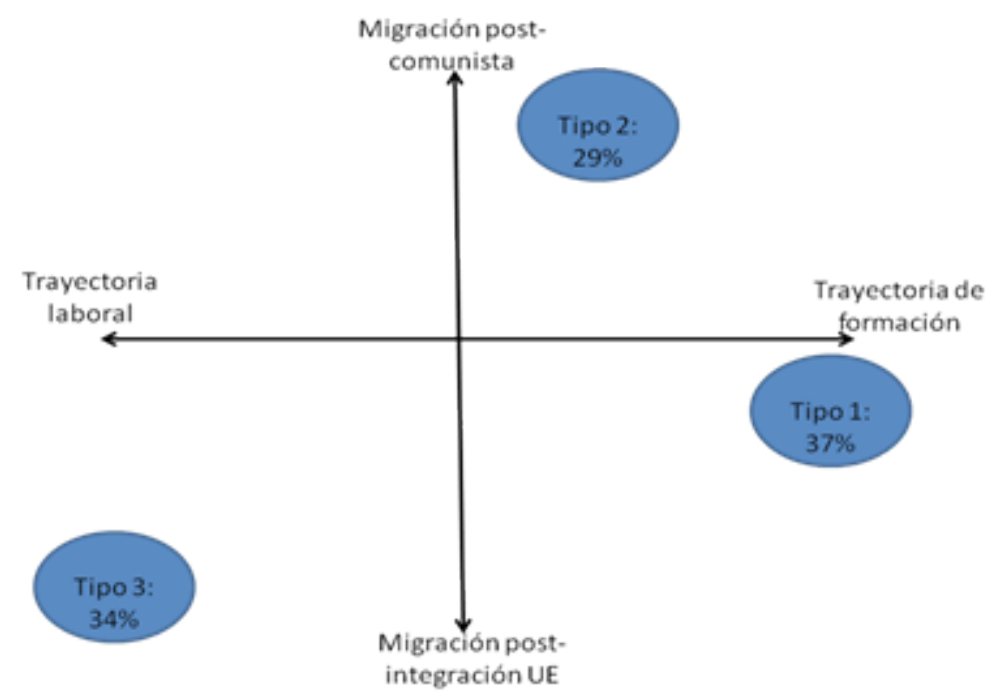

Fuente: elaboración propia en base al programa SPAD

De los tres clusters identificados, el cluster 3 ha sido escogido para representar el punto de partida de la tipología cualitativa de las entrevistas en Barcelona. Las razones para descartar los tipos 1 y 2 tienen que ver con sus propias características, y sus desajustes con los objetivos de la investigación. El tipo 1 presenta un perfil que cuenta sobre todo con trayectorias formativas en el país de destino (perfil de estudiantes internacionales). Debido a que el propósito de la investigación es la reconstrucción de trayectorias migratorias de profesionales, este cluster y sus características no resultaban útiles. Por otro lado, el cluster 2 ha sido descartado al corresponder a un perfil que emprenden proyectos migratorios en los primeros años de la transición democrática, sobre todo hacía EE.UU y Canadá.

No obstante, el tipo 3 sí refleja un tipo de emigración intra-europea. El tipo 3 (34\% de la muestra) engloba a migrantes que en su gran mayoría (69\%) han finalizado los estudios universitarios en Rumanía. El hecho de apuntar a terceros países y Rumanía como los lugares donde han adquirido los conocimientos teóricos, revela que se trata de una migración circular, ya que son personas que han experimentado varios procesos migratorios. Tal y como apunta Piracha y Vickerman (2002), en la movilidad intraeuropea la migración cualificada no es permanente, sino que las migraciones repetidas representan respuestas a una economía dinámica. En este sentido, 
la circularidad no se entiende en clave de retorno al país de origen; sino que supone movilidad entre varios países, siendo el espacio europeo propiciador de este tipo de desplazamientos.

En cuanto a la situación laboral en Rumanía, el 32\% declara haber estado trabajando en el sector privado y el $10 \%$ en el sector público. Además, el $15 \%$ declara que en Rumanía estaba empleado en la categoría de técnicos y profesionales científicos e intelectuales Senior. El 27\% de la muestra trabaja actualmente en actividades profesionales vinculadas a las telecomunicaciones; programación, consultoría y otras actividades relacionadas con la informática; servicios financieros; intermediación de comercio; actividades de investigación y desarrollo en estos ámbitos.

Entre las razones para emigrar, destaca como primera razón el hecho de ganar experiencia internacional (laboral, educativa, personal); seguida por otras razones, tales como el traslado por parte de una compañía multinacional o la contratación en origen. En relación a las razones para escoger el actual país de residencia, destaca el hecho de que allí se ubica la organización para la que trabajan, mientras que casi el $20 \%$ ha escogido el país de destino por las características del programa universitario ("era gratuito o de bajo coste", "porque fui aceptado en ese programa", "porque solo aquí se estudiaba lo que yo quería"). En cuanto al bloque referido a la participación en actividades comunitarias, los rumanos que pertenecen a este tipo, en su inmensa mayoría participan (96\%), aunque con distinta frecuencia: el $30 \%$ una vez al mes, el $18 \%$ una vez cada dos meses y el $20 \%$ una vez al año. El tipo de participación se refiere a encuentros informales con amigos y conocidos (38\%) y a eventos culturales (20\%).

\subsection{Desde trayectorias migratorias de continuidad a trayectorias de ruptura: ¿circularidad o permanencia?}

En cuanto al perfil de profesionales rumanos que miran a Barcelona a través del traslado de empresa multinacional, las razones que explican este traslado guardan relación con la oportunidad brindada por la empresa. Concretamente, la migración se entiende como un nuevo reto profesional ya que los entrevistados describen altos niveles de satisfacción con sus vidas en Rumanía. En otras ocasiones, sin embargo, las entrevistas revelan que dicho traslado es provocado como estrategia para desarrollar un proyecto migratorio "seguro" y donde se garantice la incorporación al mercado laboral cualificado. Parte de este deseo de provocar el traslado tiene que ver con un discurso crítico con respecto a las corporaciones multinacionales que operan en el país de origen. Así, muchos destacan que las condiciones laborales (nivel de remuneración, exigencias, ritmos de trabajo, intensidad y complejidad de las tareas exigidas, posibilidades de promoción) ofrecidas por las multinacionales son superiores en otros países europeos. Por ello, trabajar duro durante varios años para provocar el traslado a Barcelona, 
representa una estrategia utilizada por algunos rumanos de este perfil.

La empresa multinacional se configura como una verdadera malla de seguridad en la configuración del proyecto migratorio, capaz de generar infraestructuras privilegiadas para el migrante. La organización no demanda la homologación de los estudios universitarios y se encarga de los trámites logísticos que implican el traslado y la obtención de los permisos de trabajo. Incluso durante la imposición de las dos moratorias del gobierno español ${ }^{5}$, estos migrantes cuentan con el apoyo de la empresa que les asesora en el proceso, mediante consultoras jurídicas especializadas.

Otro elemento que explica por qué las empresas multinacionales operan como mallas de seguridad, tiene que ver con el ambiente de trabajo. La amplia experiencia de estos entrevistados y el hecho de que desarrollen tareas muy parecidas a las de las oficinas de Bucarest, son elementos relevantes a la hora de comprender su rápida adaptación en Barcelona. A este aspecto se le añade el hecho de que conocen a algunos de sus homólogos; en ocasiones están familiarizados con las oficinas de Barcelona y siguen utilizando el inglés como idioma vehicular. El testimonio de esta mujer de 28 años que llega a Barcelona en 2010 es revelador.

-¿Qué sabías sobre el mercado laboral español antes de tu llegada?

-Absolutamente nada...no sabía nada...ni siquiera me documenté por Internet sobre qué significa la vida en Barcelona, qué se puede visitar en Barcelona o cómo es la estructura de la ciudad...No tuve que preocuparme por nada porque tenía todo organizado por parte de la empresa. Sabía que iba del aeropuerto al hotel, de ahí un mes podía quedarme en un hotel y que me iban a pagar una agencia que me buscara piso así que no tenía que preocuparme por nada y en consecuencia no me impliqué más de lo necesario.

Por todo lo dicho anteriormente, el proyecto migratorio de estos migrantes se interpreta en clave de continuidad, tal y como lo manifiesta una de las entrevistadas a través del gráfico 2.

${ }^{5}$ En el año 2007, año de la incorporación de Rumanía en UE, todos los países miembros, excepto Finlandia y Suecia, imponen un periodo transitorio durante el cual se restringe la libre circulación de mano de obra rumana (excepto aquellos que residen en un país UE por fines no lucrativos, por estudios o porque están empleados por cuenta propia). En el año 2009, el gobierno español pone fin a la moratoria y los ciudadanos rumanos pasan a tener pleno acceso al mercado laboral, situación que cambia en el 2011 cuando se reintroduce la restricción. En la actualidad, los ciudadanos rumanos tienen derecho a circular y trabajar en el territorio español. 
Gráfico 2. Trayectoria migratoria perfil multinacional (mujer 34 años)

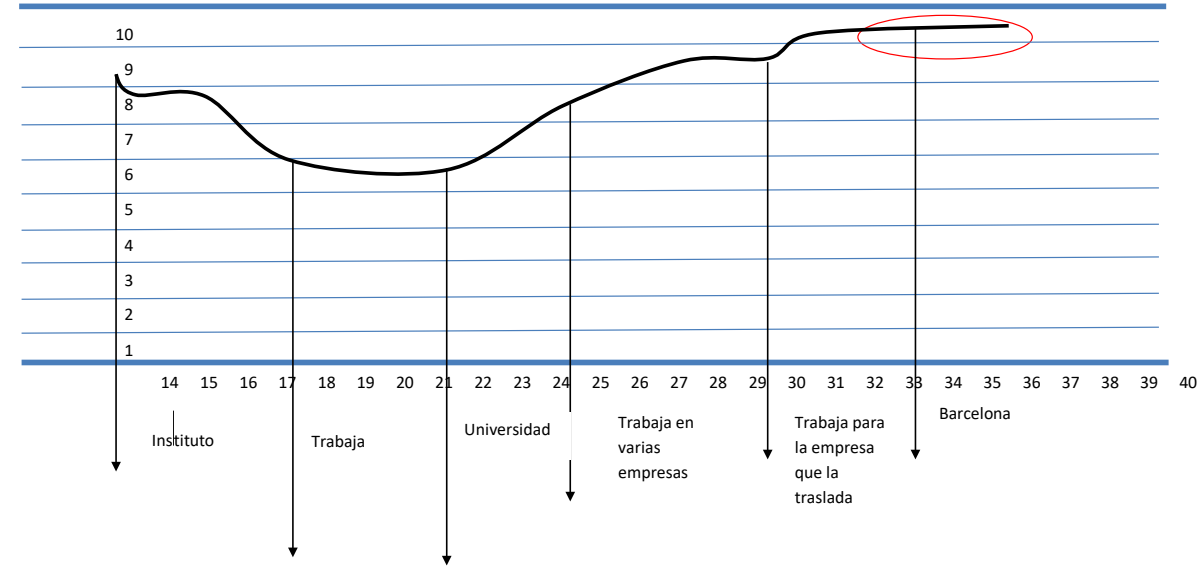

Cuando se trata de evaluar el proyecto migratorio, los entrevistados destacan haber ganado calidad de vida (las infraestructuras de la ciudad, la gastronomía, la amabilidad de la gente, el ritmo de vida, el carácter cosmopolita y la oferta de ocio). De hecho, la cuestión de la calidad de vida también aparece en la narrativa de los entrevistados pertenecientes a los otros dos perfiles. El segundo elemento positivamente valorado tiene que ver con las condiciones de trabajo, ya que tanto a nivel salarial, ritmos de trabajo u horas extra, su vida en España es percibida en clave de ganancia.

A pesar de valorar positivamente su vida en Barcelona estos migrantes no necesariamente se plantean permanecer en España a largo plazo. Esta manera abierta de entender el proyecto migratorio tiene que ver con una serie de cuestiones. Especialmente en el caso de las mujeres, el momento del ciclo vital es clave y la permanencia depende de los vínculos que los entrevistados establezcan en Barcelona. Esta cuestión aparece también en el discurso de entrevistados de los otros dos perfiles. En su entrevista Angela de 26 años comenta:

A veces pienso que tengo una edad y me gustaría tener una familia pero no tengo nada claro que quiera criar a mis hijos aquí así que lo personal marcará mi decisión de quedarme o retornar.

En segundo lugar, el proyecto migratorio se entiende de manera abierta, siendo el retorno una posibilidad que no descartan. A pesar de que, independientemente del perfil, el retorno se percibe en clave de retroceso, sobre todo a nivel salarial y en relación a la calidad de vida, para los rumanos que migran a través de traslado de multinacional retornar es una opción viable y realista. Primero, debido a que los testimonios describen una vida feliz en Rumanía, tanto a nivel personal como profesional. Además, el acuerdo de traslado incluye la opción de reincorporarse en cualquier momento a sus puestos 
de trabajo en Rumanía.

En cuanto al segundo perfil estudiado en la etapa cualitativa (migrantes rumanos con estudios de postgrado en España), corresponde en gran medida al perfil de estudiantes internacionales. Así, la decisión de continuar los estudios en el extranjero representa un valor añadido, al ser considerados más prestigiosos que en Rumanía. A la percepción según la cual el sistema universitario en Rumanía no prepara para la incorporación en el mercado laboral, se le añade la influencia del grupo de pares que realizan estudios de tercer ciclo en el extranjero y que evalúan muy positivamente esta experiencia.

Si el máster en el extranjero es el objetivo a alcanzar, ¿cuál es el razonamiento para escoger España y Barcelona como ciudad de destino? La elección de España como país de destino tiene que ver con dos criterios: 1) el precio de los estudios de postgrado y el nivel de vida relativamente más bajo que en otros países europeos; 2) la percepción de que la sociedad española es abierta y bastante cercana a la rumana. Adela de 27 años explicaba así las razones para escoger Barcelona:

Hablando con una amiga empezamos a buscar un máster y encontramos varios en Londres pero eran muy caros así que finalmente optamos por este en España. Además tenía otra amiga que estudiaba aquí en ESADE y me habló maravillas de la vida en Barcelona.

En cuanto al papel de los estudios, el trabajo de campo revela que para este perfil las titulaciones de postgrado en España representan, el trampolín para el acceso al mercado cualificado. Las instituciones privadas que imparten estos programas cuentan con amplias bolsas de prácticas remuneradas. Estas prácticas son clave en la incorporación laboral, pero también en la trayectoria legal, ya que generan un primer vínculo con el mercado cualificado, incluso durante la moratoria. En segundo lugar, es relevante el papel de las credenciales obtenidas en España en relación al significado simbólico de las mismas y la capacidad de generar redes sociales valiosas para la incorporación en el mercado cualificado. Además, a través de lazos débiles (compañeros de máster), algunos entrevistados son recomendados o tienen acceso a puestos de trabajo que les proporcionan movilidad ocupacional ascendente.

Así, la experiencia educativa y la incorporación laboral en clave de éxito, terminan influyendo de manera positiva en la evaluación del proyecto migratorio. Por ello, cabe entender las trayectorias de los rumanos de este perfil en clave ascendente (gráfico 3). 
Gráfico 3. Trayectoria migratoria perfil con estudios terciarios en España (mujer 31 años)

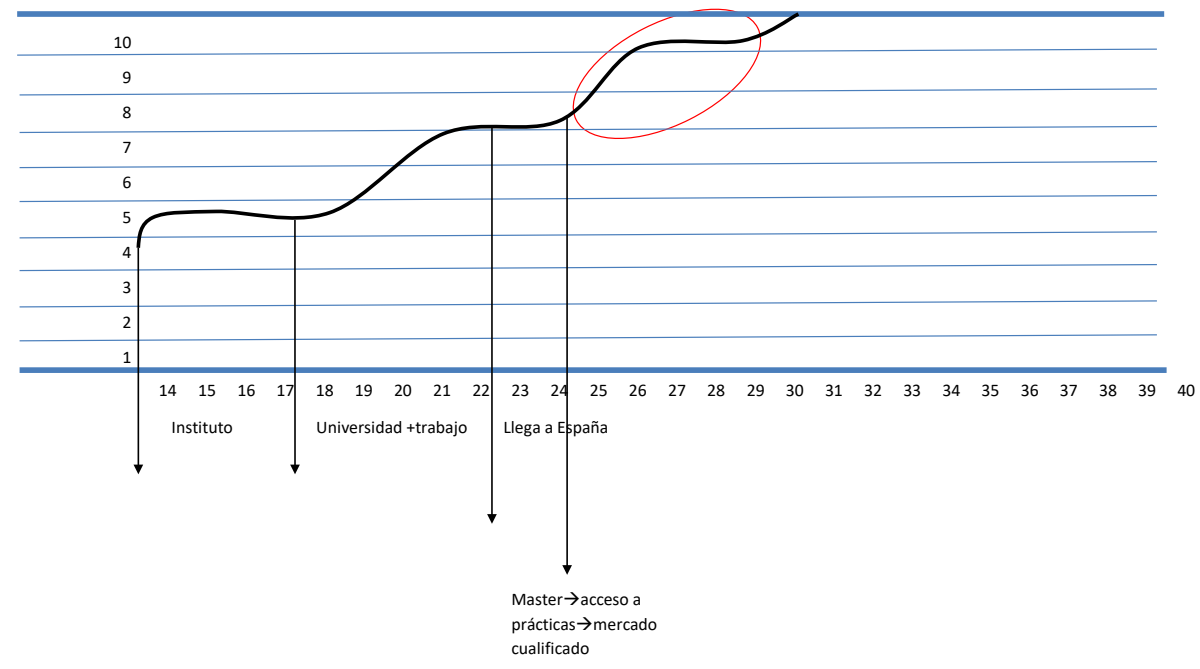

Referido a las perspectivas de futuro, la mayoría de estos entrevistados inicialmente se plantean proyectos migratorios abiertos, en los que no descartan el retorno, pero tampoco la permanencia en España. No obstante, a medida que se produce la consolidación en el mercado cualificado, el retorno es percibido como una última opción y a corto plazo se descarta. El discurso crítico con respecto a la situación en Rumanía juega un papel importante en la concepción del retorno que es visto en clave de retroceso tras haber invertido en su formación en España. Eso no significa, necesariamente que, a largo plazo, se proyecten en España tampoco. La falta de oportunidades laborales como consecuencia de la crisis les lleva a plantearse la posibilidad de emprender otros proyectos migratorios hacia terceros países europeos. Este es el testimonio de Madalin, de 26 años.

Tengo poco contacto con Rumanía, no me atrae para nada porque desde mi punto de vista es un país con poco valores no hay una apuesta por la profesionalidad, no se aprecia mucho y entonces llegué a la conclusión que no tengo nada que hacer en Rumanía y estoy convencido que incluso si evolucionara la cosa, que hasta ahora en 15 años no he visto progresos significativos para que se llegue a un mínimo aceptable...no tengo nada que hacer de vuelta a casa. Si viera en Rumanía que pudiera cumplir lo que yo deseo de mi vida profesional, probablemente estaría ahi ahora, aunque sinceramente te digo que incluso Barcelona se me está haciendo pequeña desde un punto de vista profesional...como ciudad está bien pero me doy cuenta que no hay progreso en España. 
En relación al tercer perfil estudiado, las razones para migrar guardan relación con los clásicos factores de atracción-expulsión. Entre los elementos de atracción destacan los imaginarios culturales que estos migrantes proyectan sobre España. Se trata, sobre todo, de personas que completan sus estudios terciarios en el extranjero y que en el momento de migrar a España cuentan con largas trayectorias educativo-laborales en terceros países. Sin embargo, al margen del éxito profesional en Estados Unidos, Holanda o Inglaterra son países donde los entrevistados no terminan de encontrarse plenamente adaptados. Así, a pesar de las limitaciones del mercado laboral español escogen este país tras priorizar aspectos personales. Además, gracias a sus experiencias previas y las oportunidades profesionales a las que tienen acceso, el acceso al mercado cualificado es automático.

También destacan aquellos migrantes para los cuales los factores de expulsión cobran especial relevancia. Se trata de personas cuyas trayectorias laborales en Rumanía quedan marcadas por el precario contexto económico del país. Concretamente, se trata de trabajadores del sector público (investigación y educación), afectados por los ajustes económicos aplicados durante la transición. Además de los empleados públicos, las entrevistas apuntan a otro perfil vulnerable: los jóvenes emprendedores y las personas con trayectorias de precariedad en Rumanía. Estos migrantes señalan cierto desgaste a la hora de desarrollar proyectos profesionales en un contexto que perciben como hostil para la innovación y el desarrollo, en el que predominan las prácticas de corrupción y nepotismo, una administración lenta e ineficaz y una inestabilidad que obstaculiza el desarrollo de carreras profesionales y proyectos vitales. A esta sensación de frustración que experimentan, se le añade la desconfianza en que la situación cambie a medio plazo, lo que justificaría su decisión de migrar.

Más allá de las razones para la inmigración, cabe destacar el tipo de trayectorias que desarrolla este perfil. Sin mallas de seguridad ni trampolines, para este perfil cobra significado la debilidad de las redes fuertes y la fuerza ejercitada por las redes débiles (Granovetter, 1983). Concretamente para aquellos que desarrollan su proyecto migratorio debido a factores de expulsión, contar con redes familiares en España representa una cuestión de peso en la elección del país de destino. Sin embargo, cuando la red familiar está vinculada a sectores no cualificados, su papel puede ser más bien simbólico o incluso se debilita a medida que el migrante logra la incorporación laboral acorde a su formación. Aparte de las redes familiares, destacan las redes de conocidos (excompañeros de trabajo) que recomiendan a los entrevistados para sus actuales puestos de trabajo. Este tipo de redes son fundamentales para entender el proyecto migratorio de muchos de los que pertenecen a este perfil cuyo nivel de castellano es bajo o no cuentan con credenciales en España o terceros países.

En cuanto a la evaluación del proyecto migratorio, para la mayoría de los entrevistados de este perfil, el proyecto migratorio representa un punto de inflexión. Aquellos proyectos migratorios en los que los factores de expulsión juegan un papel relevante y donde la incorporación en el mercado cualificado se produce de forma inmediata y exitosa, la migración se traduce en un punto de 
inflexión ascendente (gráfico 4). En la evaluación que estos migrantes hacen de su proyecto migratorio, un peso importante lo tiene el discurso especialmente crítico con aspectos de la sociedad rumana (corrupción, falta de transparencia, incompetencia política etc.). Si para el conjunto de los entrevistados el salto salarial en España es interpretado en clave de ascensión, para los que llegan a España como consecuencia de los factores de expulsión, esta cuestión cobra todavía mayor relevancia. Así lo ejemplifica Vlad, un programador de 26 años que llega a Barcelona en el 2007.

Aquí con mi sueldo de programador me puedo permitir muchas más cosas, mejoré mi estilo de vida, me compré ropa, un buen ordenador etc. esto es calidad de vida. Viajamos más (él y su pareja) y podemos salir a cenar... todo esto no podía hacerlo con mi sueldo en Rumanía.

Gráfico 4. Trayectoria migratoria perfil sin estudios terciarios en España (hombre 36 años)

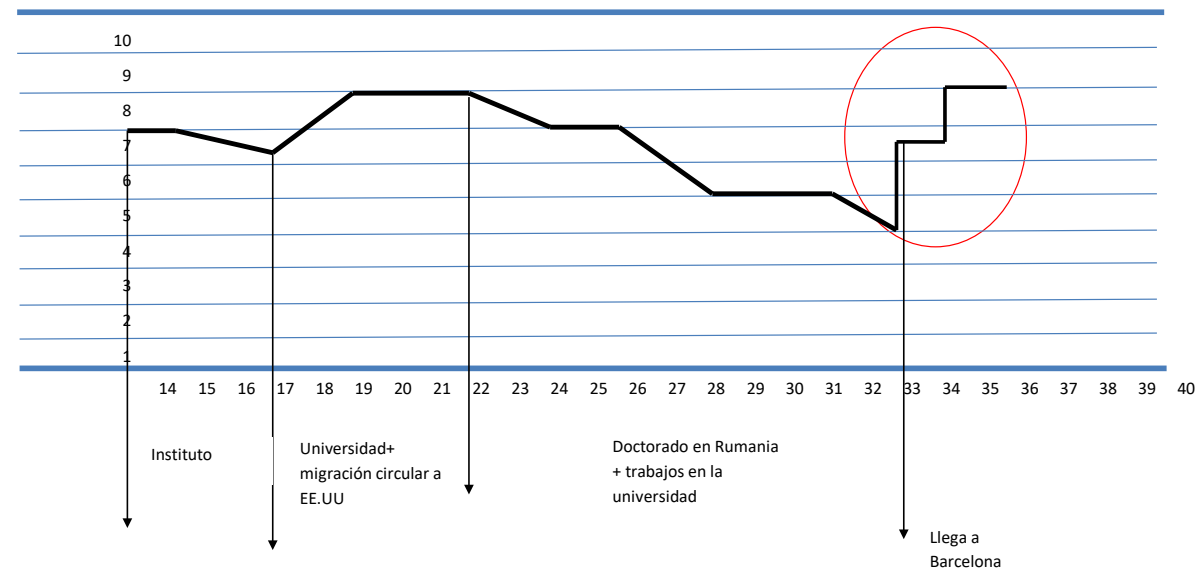

Sin embargo, no todos los que pertenecen a este perfil evalúan su proyecto en clave de punto de inflexión ascendente. Así, la falta de redes sociales o la incapacidad de las mismas para generar la incorporación al mercado cualificado provoca, en algunas situaciones, inconsistencia de estatus o periodos de inactividad y el proyecto migratorio se convierte en un punto de inflexión descendente. 
Gráfico 5. Trayectoria migratoria perfil sin estudios terciarios en España (hombre 36 años)

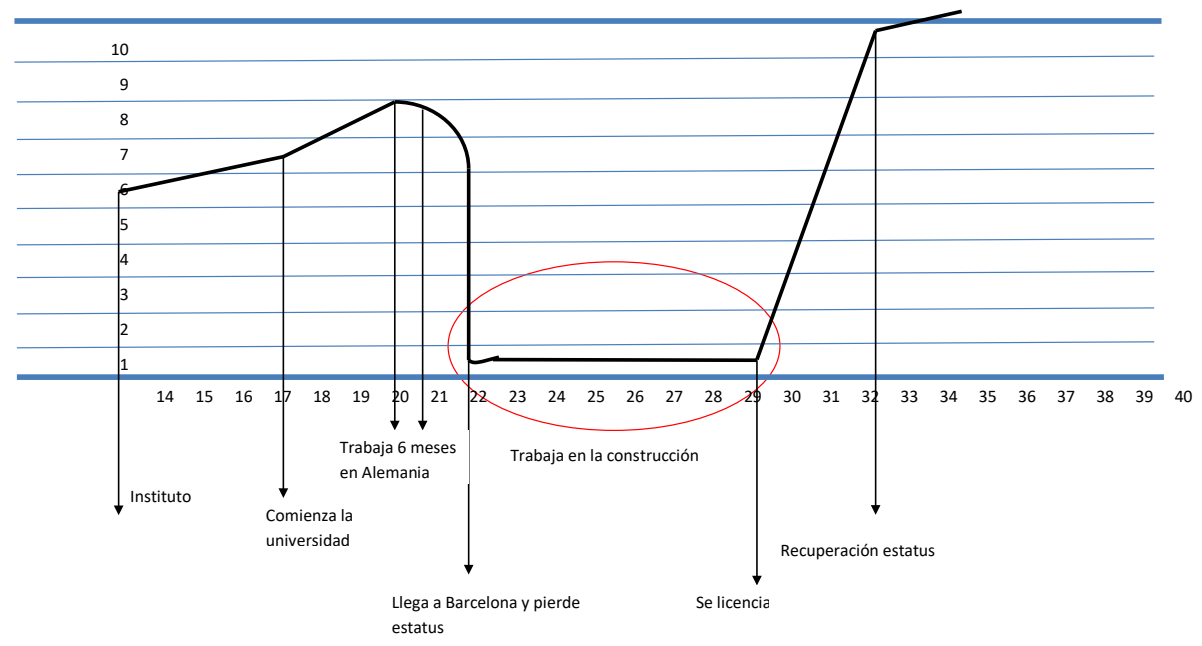

Independientemente de si los migrantes experimentan proyectos ascendentes o descendientes, lo que destaca de este perfil es su propensión a desarrollar proyectos permanentes en España, donde el retorno a Rumanía es la última opción y la menos deseada. Esto tiene que ver con una serie de cuestiones que se vinculan a las razones para migrar. En el caso de aquellos que emigran por la fuerza de los factores de expulsión, el retorno queda descartado por el discurso crítico con respecto a sus experiencias en Rumanía y por la falta de expectativas de mejora. Por otro lado, para aquéllos que han residido en varios países a lo largo de los últimos años, retornar supone un retroceso personal, en cuanto a estándares de vida.

Un ejemplo en este sentido lo representa Ion, de 36 años. En el momento de la entrevista estaba pendiente de obtener la Green Card. A la pregunta "¿dónde te ves viviendo de aquí a 5 años?", responde:

Yo me veo viviendo aquí en España para siempre...aunque haya incertidumbres con lo de la green card pero es poco probable más que nada porque mi hijo tiene 4 años, lo traje de Rumanía y fue difícil porque tuvo que aprender el idioma, adaptarse a la escuela y ahora le va bien sabe castellano y catalán y si lo llevo a otro sitio, sería demasiado para él porque sería empezar de cero...eso no.

A pesar de que estas cuestiones quedan articuladas también en el discurso de los rumanos pertenecientes a los otros perfiles, aquéllos que llevan mucho tiempo en el extranjero cuestionan elementos de la sociedad rumana que van más allá del bienestar material y se refieren a las cuestiones políticas o relacionadas con las estructuras democráticas. Así, para ellos, retornar supondría no encajar 
con unas estructuras de poder y un sistema de valores percibidos como diferentes a los que han adoptado a lo largo de sus trayectorias. Aunque exista la esperanza de una mejora en Rumanía, la mayoría de los entrevistados la postergan a un momento lejano que desincentiva el retorno.

\section{CONCLUSIONES}

El artículo explica las trayectorias migratorias de tres perfiles de inmigrantes rumanos altamente cualificados residentes en Barcelona, a través de un diseño mixto de tipo secuencial. En este sentido, a nivel metodológico dos son las implicaciones a la hora de utilizar este tipo de diseños en el estudio de las migraciones cualificadas. En primer lugar, ha resultado de suma utilidad la aplicación de la encuesta online (y el análisis de clasificación) como herramienta para determinar la selección de los perfiles estudiados en la segunda parte (cualitativa). Eso es así especialmente en casos como éste, el de los rumanos altamente cualificados, sobre los cuales contamos con pocas investigaciones y escasas estadísticas fiables. A pesar de que GRASP no es una organización que englobe exclusivamente a profesionales rumanos residentes en España, el cluster 3 revela una serie de características que muestran un perfil de rumanos que circulan en la ampliada UE y que, a pesar de la crisis económica, siguen encontrando oportunidades de trabajo en el extranjero, precisamente porque se trata de sectores de actividad de gran demanda de mano de obra. Además, los perfiles corresponden a los que recoge la literatura sobre migración cualificada (traslado multinacional, estudiantes internacionales), con lo que la elección de los entrevistados en Barcelona queda justificada tanto teórica como empíricamente. La segunda aportación metodológica del artículo guarda relación con la manera en la que se imbrican los gráficos de satisfacción vital con el análisis de las entrevistas biográficas. La utilización cualitativa de estos gráficos enriquece el análisis, en tanto que permite a los entrevistados reflexionar sobre sus propias trayectorias y los elementos que constituyen rupturas o continuidades; a la vez que generan un material complementario para el investigador a la hora de hacer confluir los hallazgos que se elaboran a partir de narrativas y material visual.Más allá de las cuestiones metodológicas, a nivel teórico el artículo destaca la necesidad de superar las concepciones clásicas de las migraciones cualificadas entendidas como fugas de cerebros. Es por ello que se trae a colación la relevancia de las redes de cerebros a la vez que se cuestiona que la circularidad sea un fenómeno que se produzca de forma automática (Petroff, 2016a). En este sentido, la heterogeneidad de perfiles que se plantean retornar, permanecer en el extranjero o reemigrar, responde a una diversidad de razones para migrar y a incorporaciones laborales distintas, dependiendo del tipo de recursos que estas personas son capaces de movilizar durante su migración. Así, mientras aquéllos que llegan a través del traslado de una empresa multinacional cuentan con una malla de seguridad que es la propia empresa, para los que responden al perfil de estudiantes internacionales, 
la institución se percibe como un trampolín que ayuda a la incorporación en el mercado cualificado, incluso en momentos de crisis económica. Finalmente, cuando los recursos de meso-nivel no existen, son los recursos de micro-nivel (redes sociales) los que explican las trayectorias de éxito. Mientras que los vínculos fuertes pueden condicionar la elección del país de destino o incluso las primeras etapas en el proceso migratorio, estos vínculos muestran su debilidad sobre todo a la hora de acceder a puestos cualificados. Por el contrario, los vínculos débiles conformados por conocidos y excompañeros son claves para explicar estas trayectorias.

Otra cuestión relevante es la necesidad de entender estos procesos migratorios desde la perspectiva de trayectoria del curso de vida. Para explicar trayectorias migratorias y proyecciones de futuro de estos migrantes, es imprescindible reconstruir las etapas formativas y laborales previas. Sin ello, difícilmente podemos establecer hasta qué punto la migración representa una ruptura con respecto a etapas previas o, por el contrario, si el proyecto migratorio debe interpretarse como una transición suave. Por todo lo dicho anteriormente, es necesario seguir estudiando el perfil de migrantes cualificados en contextos poco comunes; al igual que seguir indagando sobre este perfil específico que proviene de los países del Este de Europa, cuyas características y circunstancias históricas lo hacen especialmente interesante para el estudio.

\section{BIBLIOGRAFÍA}

ACKERS, L. (2005): "Moving People and Knowledge: Scientific Mobility in the European Union”, International Migration, vol. 43, pp: 99-131.

ALEXE, I. el al., (2011): Al patrulea val. Migratia creierelor pe ruta RomaniaOccident Fundatia Soros Romania.

BAUER, M., et al. (2000): "Quality, quantity and knowledge interests: avoiding confusions", en Qualitative researching with text, image and sound: a practical handbook escrito por Martin W. Bauer, George Gaskell, Sage Publications.

BENSON-REA M. y RAWLINSON S. (2003): "Highly Skilled and Business Migrants: Information Processes and Settlement Outcomes", International Migration vol. 41 (2).

BRANNEN, J. (2004): "Working qualitatively and quantitatively" en Qualitative Research Practice coordinado por Clive Seale, Giampietro Gobo, Jaber Gubrium, David Silverman.

CACHÓN, L. (2002): "La formación de la España inmigrante: ciudadanía y mercado", Revista Española de investigaciones Sociológicas, 97, pp: 97-126.

CARR, S. et al. (2005): "From global careers to talent flow: Reinterpreting brain drain", Journal of World Business vol. 40 (2005), pp: 386-398.

CIUMASU, M. (2007): "International circulation of talent - a solution for the development of Romania", University within Society - UNISO, University of Versailles Saint-Quentin-en-Yvelines.

CRESWELL, J. W. (2003): Research Design: qualitative, quantitative, and mixed methods approaches (2. ${ }^{\mathrm{a}}$ ed.) Londres: Sage. 
CSEDO, K (2009): New Eurostars? The labour market incorporation of East European professionals in London, tesis doctoral, London School of Economics.

DOMÍNGUEZ, M. y LOPEZ, P. (1996): "La construcció de tipologies: procés i técniques d'análisi de dades", Papers. Revista de Sociologia, no. 48: 31.39.

FERRO, A. (2008): A picture of the highly skilled labour migrations from Romania, tesis doctoral, Universidad Biccoca Milano.

GAILLARD, J., y GAILLARD A. M. (1997): "Introduction: The International Mobility of Brains: Exodus or Circulation?" Journal of ScienceTechnology Society, $195-228$.

GASKELL, G. (2000): "Individual and group interviewing”, en Bauer, M. W. y Gaskell, G.: Qualitative researching with text, image and sound: a practical handbook.Sage Publications.

GHATAK, L. (2004): “US-Bangalore-US: IT Pros Get Cultural Jet Lag”, Economic Times, 15 July, disponible online www.economictimes.indiatimes.com.

GÓMEZ VENTURA, G. (2013): Highly Skilled Indian Migrant Population in Spain CARIM INDIA - Developing a knowledge base for policymaking on India-EU migration Co-financed by the European Union, European University Institute, available online at http://www.india-eu-migration.eu/media/CARIM-IndiaHSM-2013-39.pdf, reviewed on November 2014

GRANOVETTER, M. (1983): "The strength of weak ties: a network theory revisited", Sociological Theory, (1), pp: 201-233.

HANNAM, K, SHELLER M. Y URRY, J. (2006): "Mobilities, immobilities, and moorings". Mobilities 1(1): 1-22.

HORVAT, V. (2004): "Brain Drain. Threat to Successful Transition in South East Europe?" Southeast European Politics, vol. 5(1), pp: 76-93.

IREDALE, R. (2001): "The migration of professionals: theory and typologies." International Migration, vol. 38 (5), pp: 7-24.

KHANDRIA, B. (2001): "Shifting paradigms of globalization: the twentyfirst century transition towards generics in skilled migration from India" International Migration, vol. 39, pp: 45-71.

MARCU, S. (2007): "Dinámica y estructura migratoria laboral de rumanos: 19902006. Flujos de emigración hacia España, destino Madrid”, Revista Migraciones, (21), pp: 115-157.

MARCU, S. (2015): "Uneven mobility experiences: Life-strategy expectations among Eastern European undergraduate students in the UK and Spain", Geoforum, 58: 68-75.

MARCU, S. (2013): "Entre migración y movilidad: prácticas de movilidad transfronteriza de los europeos del Este hacia España." Revista de Estudios Europeos, $\mathrm{n}^{\circ}$ 62, p. 35-56.

MALLIMACI, F. y GIMÉNEZ, B. V. (2006): "Historias de vida y método biográfico" en Estrategias de Investigación cualitativa, Barcelona, Gedisa

MEYER, J.B. (2001): "Network approach versus brain drain: Lessons from the diaspora." International Migration (39), pp: 91-108.

MEYER, J.B. (2003): Policy implications of the brain drains changing face, SciDev. Net Policy Brief, May, www.scidev.net/dossiers/index.cfm?fuseaction=policyb rief \&dossier $=10 \&$ policy $=24$

MORSE, J. M. (2003): Principles of mixed methods and multimethod research design. In A. Tashakkori \& C. Teddlie (Eds.), Handbook of mixed methods in social \& behavioral research (pp. 189-208). Thousand Oaks, CA: Sage. 
PATTERSON, R. (2005): “U.S. Diasporas and Their Impacts on Homeland Technological and Socioeconomic Development: How Does Sub-Sahara Africa Compare?", Perspectives on Global Development and Technology, vol. 4(1), pp:83-123.

PAJARES, M. (2009): Inmigración y mercado de trabajo informe publicado por el Observatorio Permanente de la Inmigración, Ministerio de Trabajo e Inmigración.

PARELLA, S. (2003): Mujer, inmigrante y trabajadora: la triple discriminación. Barcelona, Anthropos

PETROFF, A (2013): Las trayectorias laborales de los inmigrantes rumanos cualificados en Barcelona, tesis doctoral, disponible en: http://www.tdx.cat/ handle/10803/117189

PETROFF, A (2016a): "Reversing the brain drain: evidence from a Romanian brain networking organization", International Migration, Vol. 53, Iss.5.

PETROFF, A (2016b): "Turning points and transitions in the migratory trajectories of skilled Romanian immigrants in Spain", European Societies, Vol. 18, Iss. 5.

PIRACHA, M. y VICKERMAN, R. (2002): “Immigration, Labour Mobility and EU Enlargement." University of Kent Department of Economics Discussion Paper No. 0209. http://ssrn.com/abstract=375100 or http://dx.doi.org/10.2139/ ssrn.375100 (consultado el 17 de abril 2013).

REYNERI, E. y FULLIN, G. 2008. "New immigration and labour markets in Western Europe", Transfer. European Review of labour and research, 14(4), pp. 573-588.

SOLE, C. et al. (2016): Impact of Circular Migration on Human, Political and Civil Rights, Springer, ISBN 9783319288963

STECKLER, A. et al. (1992): “Toward Integrating Qualitative and Quantitative Methods: An Introduction" Health Education and Behavior, 19, pp: 1-8.

VERD, J.M. Y LÓPEZ, P. (2008): "La eficiencia teórica y metodológica de los diseños multimétodo", Empiria. Revista de Metodología de Ciencias Sociales, 16: pp. 13-42.

VIRUELA, R. (2007): "Migración y nuevas tecnologías de la información y la comunicación: inmigrantes rumanos en España”. Migraciones, 21, pp: 259-290. 